\title{
MASTER
}

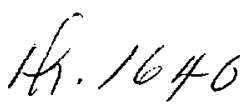

UCRL 52323

\section{THE EQUATION OF STATE}

\section{C: BERYLLIUM}

H. Graboske

L. Wong

September 15, 1977

Prepared for U.S. Energy Research \& Development Administration under contract No. W-7405-Eng-48

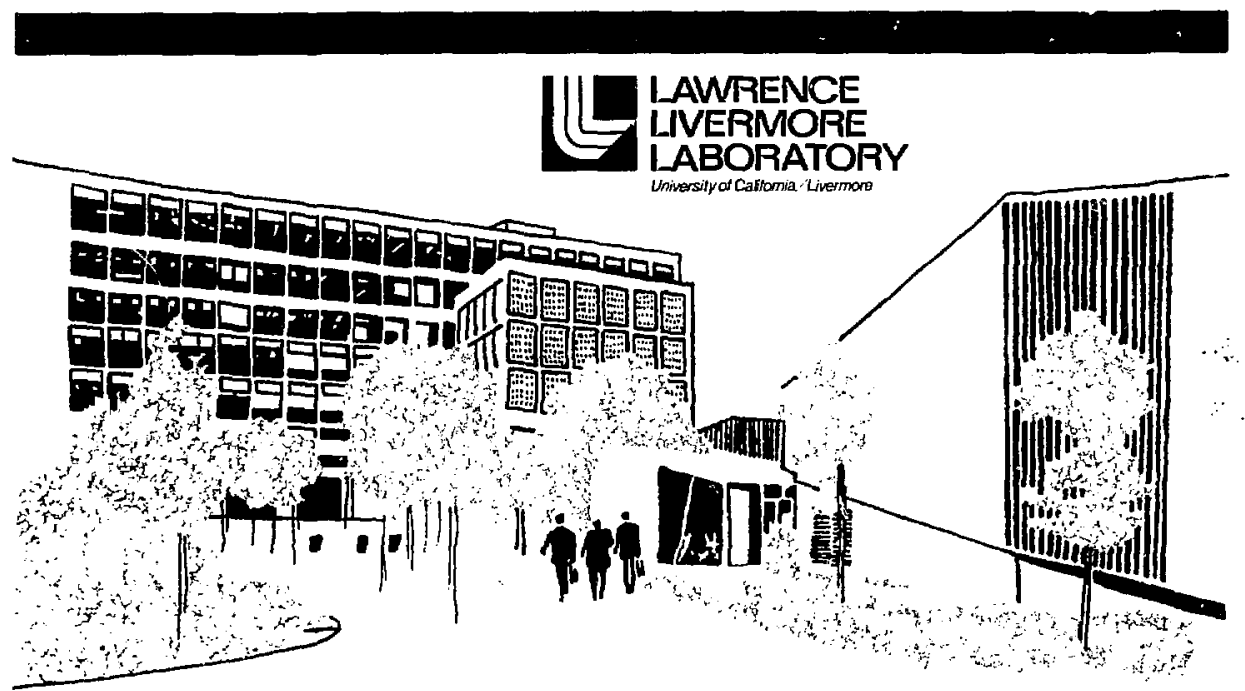




\section{NOTICE}

"This report wie prepured as an account of work sponsored by the United States Government. Nelther the United Siutes nor the United States Department of Energy, nor eny of their employees, nor any of their contractors, subcontractor, or their omployees, makes any warranty, express or implied, or assumes any legal lisbility or responsibility for the eccuracy, completeness ot usefulnes of any information, npparatus, product or process disclosed, or represents that its use would not infringe privately-owned rights."

\section{NOTICE}

Reference to a company or produce name does not imply approval or recommendation of the product by the University of California or the U.S. Department of Energy to the exclusion of others that may be suitable.

Printed in the United States of America Available from

Nationat Technical Information Service

U.S. Department of Commerce

5285 Port Royal Road

Springfield, VA 22161

Price: Printed Copy $\$$ : Microfiche $\$ \mathbf{\$ 3 . 0 0}$

\begin{tabular}{|c|c|c|c|}
\hline Page Range & $\begin{array}{c}\text { Domestic } \\
\text { Price } \\
\end{array}$ & Page Range & $\begin{array}{c}\text { Domestic } \\
\text { Price } \\
\end{array}$ \\
\hline 001025 & $\$ 4.00$ & $326 \cdot 350$ & $\$ 12.00$ \\
\hline $026 \cdot 050$ & 4.50 & $351 \cdot 375$ & 12.50 \\
\hline 051.075 & 5.25 & $376 \cdot 400$ & 13.00 \\
\hline $076-100$ & 6.00 & 401425 & 13.25 \\
\hline 101125 & 6.50 & $426 \quad 450$ & 14.00 \\
\hline $126 \quad 150$ & 7.25 & $451-475$ & 14.50 \\
\hline $151 \quad 175$ & 8.00 & $476-500$ & 15.00 \\
\hline 176 & 9.00 & $501-525$ & 15.25 \\
\hline $201-325$ & 9.25 & $526 \quad 550$ & 15.50 \\
\hline $226 \cdot 250$ & 9.50 & $551-575$ & 16.25 \\
\hline 251.275 & 10.75 & $576 \cdot-600$ & 16.50 \\
\hline $276 \quad 300$ & 11.00 & 601 up & -1 \\
\hline $300-375$ & It .75 & & \\
\hline
\end{tabular}

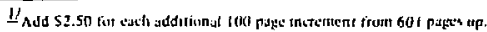




\section{近 \\ LAWRENCE LIVERMORE LABORATORY}

University of Caftornia Livermore,California 94550

UCRL 52323

\section{THE EQUATION OF STATE OF BERYLLIUM}

H. Graboske

L. Wong

MS. date: September 15, 1977

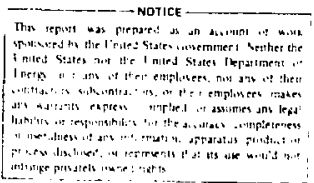

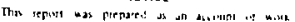

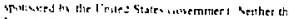

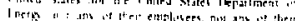

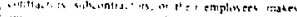

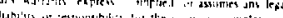

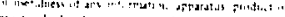

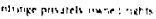




\section{THE EQUATION OF STATE \\ OF BERYLLIUM}

\section{Abstract}

A new, wide-range equation of state (EOS) has been constructed for $\mathrm{Be}$. The composite theoretical model incorporates ionization equilibrium and condensed-matter and multiphase physics. It also satisfies all thermodynamic equilibrium constraints. The theoretical EOS has been compared with all available high-pressure and high-temperature Be data, and satisfactory agreement is generally achieved. The most interesting feature is the theoretical prediction of melting at just below $220 \mathrm{GPa}$ (2 $\mathrm{Mb})$, indicating an extremely wide pressure range for solid Be. A striking feature is the appearance of she11structure effects in physical-process paths: 2 large loops appear on the principal Hugoniot and the behavior of release isentropes from $p=\rho_{0}$ is significantly affected.

\section{Introduction}

Beryllium is an important elemental metal used for a wide range of laboratory purposes, such as in LLL weapons programs. An hcp metallic solid with a high yield strength, it is unirise as a low-Z metal. Its atomic structure and phase diagram are simple. The atomic structure is shown in Table 1. In the phase diagram, ${ }^{2}$ the $\alpha$ (hcp) phase undergoes a solid-solid transition to $\beta$ (bcc) imme:]iately prior to melting at $1551 \mathrm{~K}$.

The atomic structure of Be has some very interesting thermodynamic properties, as a result of two electron shells, equaljy populated jut posessing a ten-fold difference in mean shell energy. The high first-ionization potential and the absence of a molecular state helps simplify the transition at lower densities from a multiphase metallic fluid to the plasma.

Table 1. The atomic structure of beryllium.

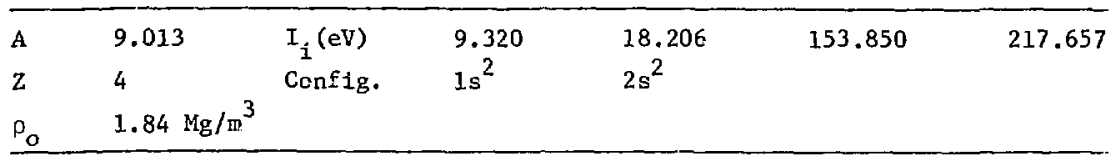




\section{Physics of the Primary EOS Codes}

To calcuiate theoretical EOS, over the range of 1000-fold expanded density to 1000-fold compressed density from room temperature $(0.02585 \mathrm{KeV})$ to $25 \mathrm{keV}$, we chose the best available theoretical model for each regime in the $T, \rho$ range, and apply this model until it breaks down or becomes inaccurate. Typically, the entire equilibrium EOS surface must be handled as at least three separate regions: the ionization/dissociation equilibriun region (rigorous in the limit $T+\infty$, or $\rho+0$ ), the condensed matter region (asymptotically exact as $T+0$ or $\rho \rightarrow \infty$ ), and the multiphase or chemical-physics region, which includes the near-normal density, low-temperature resion. The theoretical models may be first-principal calculations (stich as the coulomb gas-perturbation theory), asymptotic theories (Thomas-Fermi and other statistical atom models) or phenomenological models (normalized from experimental data).

The first difficulties inherent in this approach are encountered in constructing the composite EOS. The theoretical models may not interface well because they all tend to be asymptotically correct at widely differing extremes in the $T, \rho$ range.
This means they a11 become inexact as they approach the "central" or overlapping reglons of $T$, $p$ range. Therefore, disagreements between models may tend to maximize just where they overlap. This leads to the deletion of portions of the EOS subspaces. leaving gaps, called interpolation regions in the composite surfaces, where the various models must be joined numerically. The object of EOS theoretical studies is to reduce these gaps to a minimum and to eventually eliminate them completely.

More difficulties occur in the attempt to satisfy all fundamental thermodynamic constraints appropriate to equilibrium systems for the composite EOS. These constraints include the following conditions:

- All EOS functions must be continuous.

- Thermodynamic stability must be satisfied $\left(C_{v}, \beta_{T}\right.$ positive).

- Thermodynamic consistency must be satisfied [ $(\partial \mathrm{P} / \partial \mathrm{T})_{\mathrm{v}}$ $\left.=(\partial S / \partial v)_{T}\right]$.

We monitor two additional constraints, called normality constraints since almost al1 "normal" equilibrium 
systems obey them. They are

$$
\begin{aligned}
& (\partial P / \partial T)_{v}>0, \\
& \left(\partial^{2} P / \partial \varphi^{2}\right)_{T}>0 .
\end{aligned}
$$

Meeting a11 of these restrictions, especially for the non-physical or numerical portions of the surface (the interpolation regions), is a demanding numerical task because of the complex temperature and density behavior of a modern quantum statistical mechanical EOS calculation. That is, the shell-structure regions (thermal and pressı ionization zones, and dissociation zones) have complicated rapidly varying thermal structures as do the multiphase, chemical-physics regions with their large gradients and discontinuous derivative behavior.

For $\mathrm{Be}$, the ionization-equilibrium (IE) region was computed with the OCCIPITAL code of Rouse, ${ }^{2}$ which calculates the statistical mechanics of partially ionized mixtures with a modified Saha method. Rouse has added first order coulomb corrections (the Debye-Hückel model) in a consistent manner to the Saha ionization equilibrium and the EOS model. In addition, he computes a complete excited-state sum using the Planck partition function. The model. is the simplest extension of coulomb fluj.d theory beyond the non-interacting, partialionization method of Saha. It is accurate over a somewhat wider $T, \rho$ range than the idea1-plasma model, but becomes increasing1y inaccurate with the oniset of strong coulomb interactions, electron degeneracy, electron exchange, and correlation effects. The $T$, $p$ range covered by calculations using this model is 0.5 to $2.5 \times 10^{4} \mathrm{eV}, 10^{-3}$ to $1.84 \mathrm{Mg} / \mathrm{m}^{3}$. The condenred-matter portion of the Be EOS surface was computed using the TFCMIX code, ${ }^{3}$ The finitetemperature Kirzhnits modification of. Thomas-Fermi statistical atom theory, which includes exchange and quantum corrections to order $\mathrm{h}^{2}$ in the statistical atom framework, has been incorporated into this code. The ion fluid contribution to the thermodynamic properties is modeled by a plasma form, a Debye-Hükel weak screening limit, joined to a BrushSahlin-Teller Honte Carlo classica1ion intermediate screening model, with a freezing transition at $T=21.6$ to a harmonic Debye siolid. This theoretical model was used in the $T, \rho$ range from 0.02555 to $2.5 \times 10^{4} \mathrm{eV}$ for $1.845 \leq \rho\left(\mathrm{Mg} / \mathrm{m}^{3}\right) \leq 2000$. It then interfaces with the IE EOS over the range 1.84 to $10 \mathrm{Mg} / \mathrm{m}^{3}$ from 0.5 $\leq \mathrm{T}(\mathrm{eV}) \leq 2.5 \times 10^{4}$.

The third region, multiphase, is the most difficult to compute in 
Table 2. Be input data for GRAY.

\begin{tabular}{llll}
$\overline{\mathrm{A}}$ & 9.012 & $\mathrm{~T}_{\mathrm{m}}$ & $1551 \mathrm{~K}$ \\
$\rho_{\mathrm{o}}$ & $1.845 \mathrm{Mg} / \mathrm{m}^{3}$ & $\Delta \mathrm{S}$ & $9.637 \times 10^{-5}$ \\
$\mathrm{c}$ & $0.799 \mathrm{~mm} / \mathrm{\mu s}(7.99 \mathrm{~km} / \mathrm{s})$ & $\mathrm{g}_{\mathrm{e}}$ & $2.23 \times 10^{-\mathrm{g}}$ \\
$\mathrm{s}$ & $1.124 \mathrm{~cm} /{ }^{\mu} \mathrm{s}(11.24 \mathrm{~km} / \mathrm{s})$ & $\mathrm{E}_{\mathrm{coh}}$ & $0.266 \mathrm{Mbar}-\mathrm{cm}^{3} / \mathrm{g}=\left(2.4 \frac{\mathrm{Mbar}-\mathrm{cm}^{3}}{\mathrm{~mole}}=26.6 \frac{\mathrm{MJ}}{\mathrm{Kg}}\right)$ \\
$\gamma_{\mathrm{G}}$ & 1.25 & $\mathrm{~b}_{\mathrm{v}}$ & $0.27 \mathrm{i}$ \\
$\mathrm{a}$ & 1.46 & $\gamma_{\mathrm{e}}$ & $2 / 3$ \\
\hline
\end{tabular}

that it requires a carefully selected set of input data and then a series of iterative calculatlons leading to a best match with all available experinental data. The theoretical vehicle for this procedure is the GRAY code, ${ }^{4}$ a complex, multiphase equilibrium code designed for metals. The basic physics components of GRAY for this application include a DugdaleMcDonald form of the Gruneisen solid model, with the experimental Hugoniot daca serving as the Gruneisen reference state. The electron contribution is represented by a modified free electron gas. To this is added a Lindemann-law melting transition and a scaling- model hot-11quid regime. At Jower densities, this is smooth?y joined to a hard-sphere tlid modei. The resultant system tieats the full 1 three-phase region, with explicit treatment of the $\mathrm{L}-\mathrm{S}, \mathrm{L}-\mathrm{V}$, and $\mathrm{S}-\mathrm{V}$ two-phase coexistetice regions. The necessary input data used for Be are given in Table 2. The GRAY calculations were carried out over the range $10^{-3} \leq \rho\left(\mathrm{Mg} / \mathrm{m}^{3}\right) \leq 7$ and 0.02585 $\leq T(e V) \leq 2$. This $T, \rho$ subspace overlaps the TFC region in the range $0.02585 \leq \mathrm{T}(\mathrm{eV}) \leq 2$ at $\rho=7 \mathrm{Mg} / \mathrm{m}^{3}$, and overlaps the low-T end of the IE region over the range $10^{-3} \leq \rho\left(\mathrm{Mg} / \mathrm{m}^{3}\right)$ $\leq 1.845$.

\section{EOS Assembly}

The assembly of these three subspaces into a single composite EOS is performed by various data-handling codes. The limits chosen for the various regions are rather arbitrary, a combination of estimates of model breakdown, plus the need to reach across to join to the other theoretical models. Since internal-validity criteria are not necessarily accurate in denoting theoretical inaccuracies, the degree and nature of disagreements between the various models at their interfaces can provide Insight when 
assessing the quality of the calculations. With this in mind, let us examine tise interface detajls of the three surfaces.

An instructive representation for this purpose is the "thermal" energy surface. This is the internal-energy EOS surface plotied as a function of $\rho$ with $\mathrm{T}$ as a parameter (Fig. 1). This thermal energy Eth is defined by

$E_{t h}\left(T_{i}, \rho_{j}\right)=E\left(T_{i}, \rho_{j}\right)=E\left(T_{1}, \rho_{j}\right)$,

where $E\left(T_{1}, \rho_{j}\right)$ is called the cold curve, meaning the lowest temperature isotherm on the EOS surface. The subtraction of this component from the total surface remove. the strong density dependence appearing at $\rho>C_{0}$ of the degenerate electron gas, where $E_{e} \propto \rho^{2 / 3}$. The resultant Eth surface has a "linearized" appearance for al]. $T$, $\rho$, since the remaining energy contributions are dominated by the classical ion and thermal electron components which are approximately linear in T. A comparison of Figs. 1 and 2 shows this graphically. The advantage of the energy surface over the pressure surface is that all of the thermal structure, such as ionization, pressure ionization, melt, and $L-V$ coexistance regions, are more clearly identifiable.

The overlap between the condensed matter and multiphase regions is slown in Fig. 1, where TFC data is used for $\rho \geq 10 \mathrm{Mg} / \mathrm{m}^{3}$ and all $\mathrm{T}$, while GRAY data extends from $\rho \leq$ $7 \mathrm{Mg} / \mathrm{m}^{3}$ for $\mathrm{T} \leq 2 \mathrm{eV}$. Thit only modification required to the output EOS data was that the TFC energies be shifted down by $0.266 \mathrm{Mbar} \cdot \mathrm{cm}^{3} / \mathrm{gm}$ (26.6 MJ/kg) because the TF calculations are relative to the free atom and, thus, must be corrected by the cohesive energy of the solid. When this is done, the TFC and GRAY pressure and energy surfaces join arcurately, with the exception of a small region just above $2 \mathrm{eV}$ near $\rho_{0}$ where the TFC isotherms had to be deleted, appearing as a gap in Fig. 1. The excellent agreement between TFC and GRAY calculations is probably fortuitous. The fundamental differences in these two models usually require a renormalization of the entire TF region, a procedure less severe than it sounds, since the basic TF models 3TE rigorously correct only at pressures of 1000 Mbar (100 TPa) and higher.

The second interface, between the multiphase and IE regions, can be seen in Fig. 1 also. Here the shell structure of the IE is evident, as is the multiphase structure, both melt and vaporization, of the chemicalphysics region. The original juncture point shown here was the $2-\mathrm{eV}$ isotherm, and it is clear from the figure this 


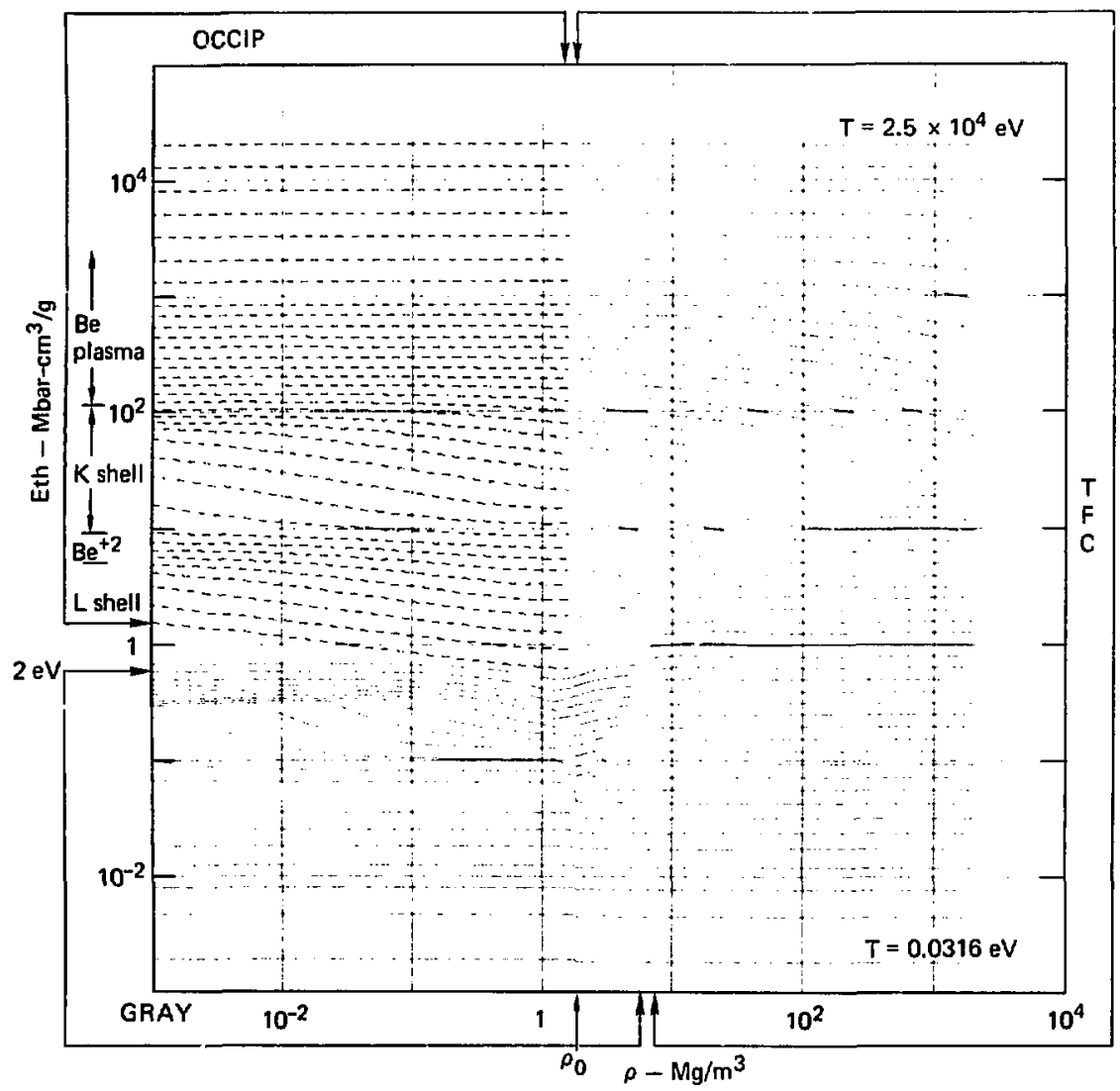

Fig. 1. The thermal energy ${ }_{4}$ surface, $E\left(T_{i}, \rho\right)-E\left(T_{1}, \rho\right)$, vs density for all $\mathrm{T}$ from 0.0316 to $2.5 \times 10^{4} \mathrm{eV}$. The three theoretical subspaces are indicated.

was not adequate because the Be fourth electron was not fully recombined at $2 \mathrm{eV}$ and low density. This association effect is evident from the decreasing energy of the lowest OCCIP isotherm $(3 \mathrm{eV})$ when compared to the filat ( $\rho$ independent) ideal, neutral atomic-gas behavior of the upper GRAY isotherm $(2 \mathrm{eV})$. Because of this, a new interface, shown in Fig. 3., was chosen, : and both models were compared over the range 0.5 to $2 \mathrm{eV}$. At that range, the OCCIP energies show that recombination of $\mathrm{Be}^{+}$to a neutral $\mathrm{Be}$ atom 


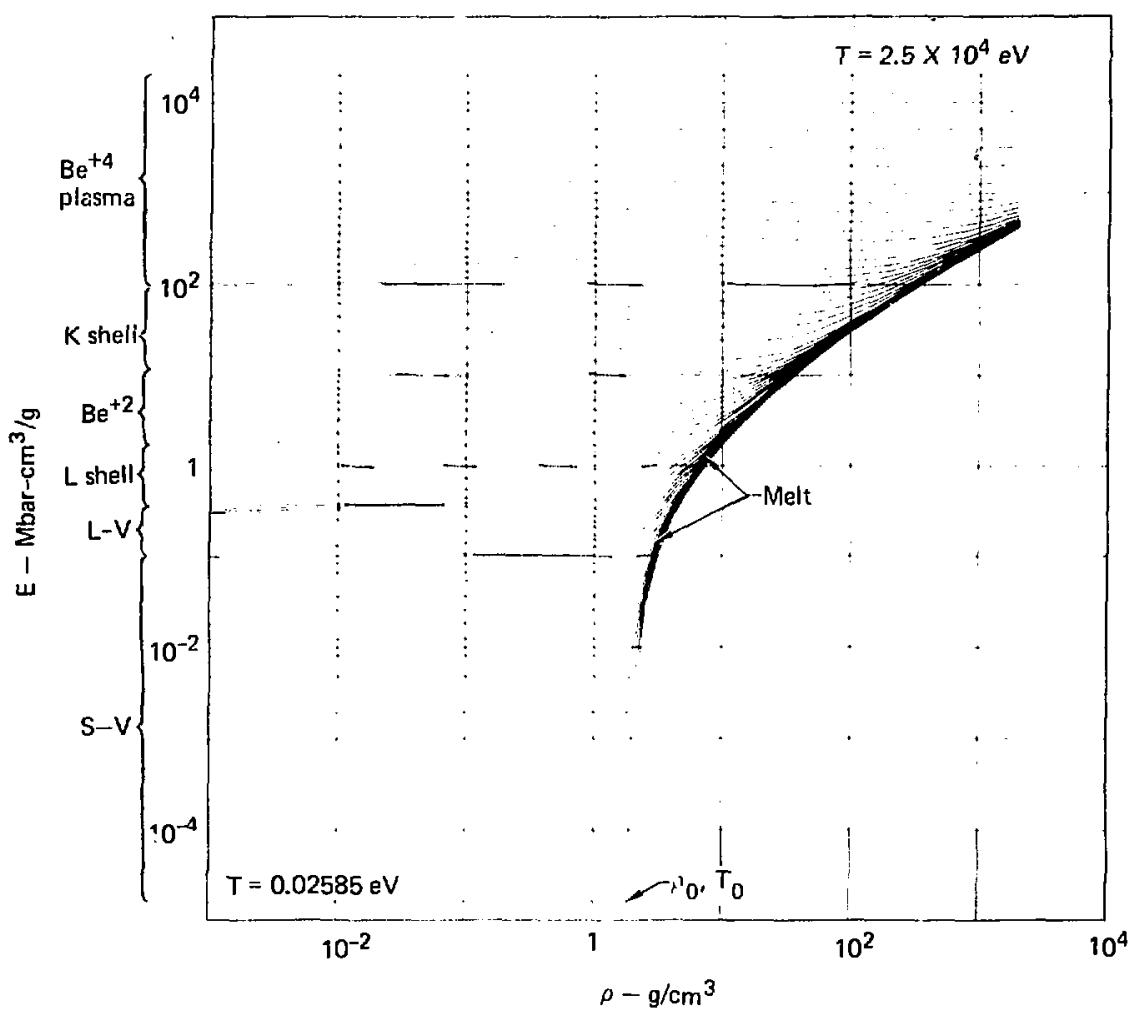

Fig. 2. The final Be energy EOS, plotted vs density. The basic $T$ anc $p$ independent variable grid in 10 points per decade, approximately logarithmically spaced. This corresponds to the sequence $1,1.25,1.6,2,2.5,3.16,4,5$, $6.3,8$, for each decade, with $P_{0}$, and $300 \mathrm{~K}(.02585 \mathrm{eV})$ as special values.

gas is only complete at $0.5 \mathrm{eV}$ (for $10^{-3}$ to $1.845 \mathrm{Mg} / \mathrm{m}^{3}$ ). At this temperature GRAY is also predicting similar, ideal neutral-fluid Be energies $\left(10^{-3}\right.$ to $\left.10^{-2} \mathrm{Mg} / \mathrm{m}^{3}\right)$. The new OCCIPGRAY interface is dropped to $0.5 \mathrm{eV}$, giving a smooth, accurate overlap of $P$ and $E$ at low density $\left(10^{-3}\right.$ to $\left.10^{-2} \mathrm{Mg} / \mathrm{m}^{3}\right)$. A problem does appear, however: in examining this new interface for $\rho>10^{-2}$ at low $\mathrm{T}$, the nonideal corrections to the ideal neutral Be gas become significant (at 0.5 and $0.63 \mathrm{eV})$, and, at about $0.1 \mathrm{Mg} / \mathrm{m}^{3}$, the GRAY model predicts the transition to the $L-V$ two-phase condensation. clearly, there is no way to join OCCIPITAL, which completely igncres 


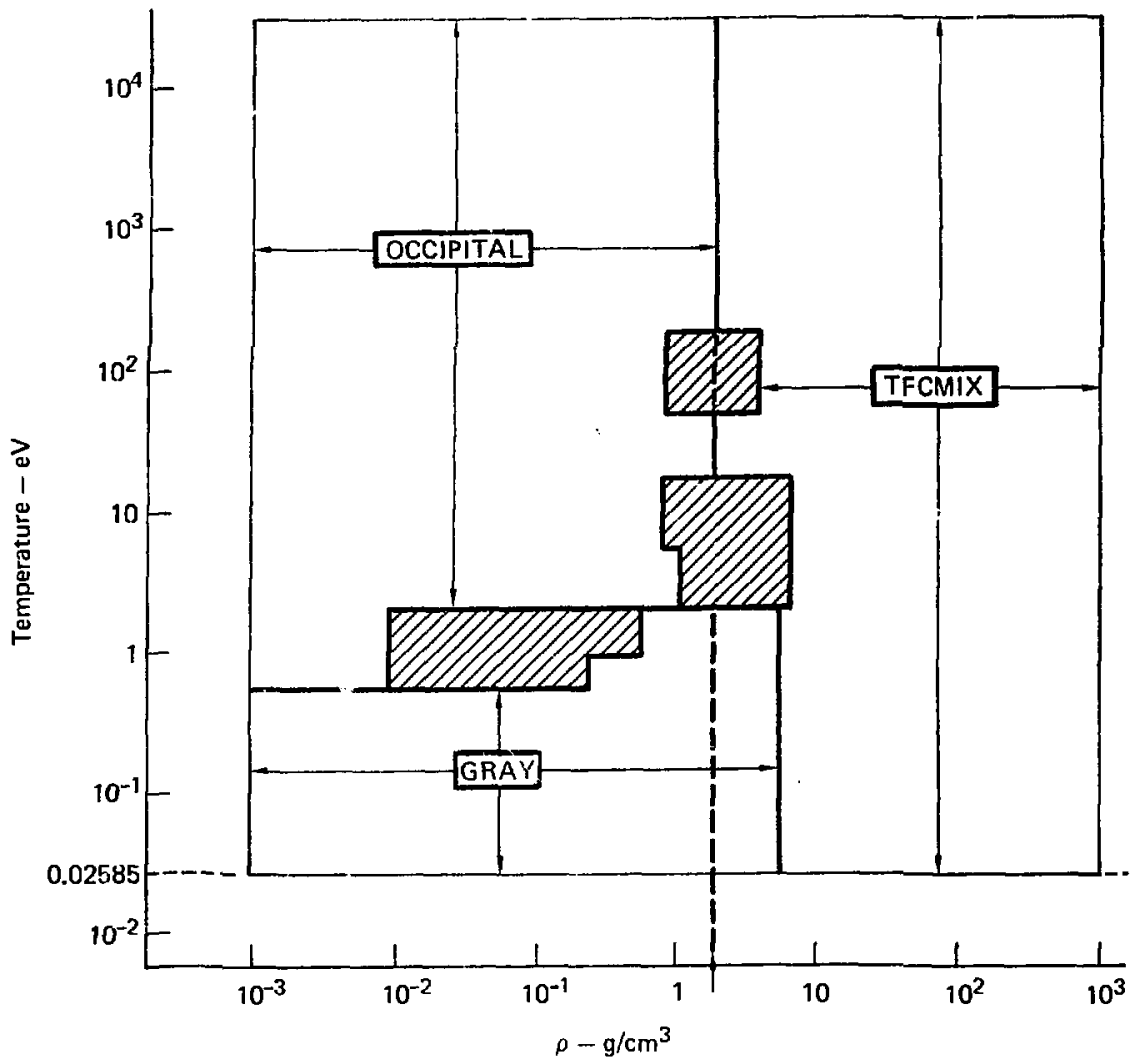

Fig. 4. The $T, \rho$ independent variable space for the Be EOS calculations. The subspaces covered by the various theoretical codes are indicated, and the shaded regions denote the interpolation regions where adjustments have been made to interface the codes together.

complexity, which is currently theoretically intractable. The development of a dense plasma-chemj.cal physics theory is clearly desirable to properly treat this region.

The third interface, between the IE and the TF regions, is fairly extensive, ranging from $2 \mathrm{eV}$ to $25 \mathrm{keV}$, at. $\rho=\rho_{0}$. The shell structure in the $1 E$ region of $F i g .1$ shows a fully fonized Be plasma for $\mathrm{T} \geq 100 \mathrm{eV}$ (at low density), a k-shell recombination zone for $100 \mathrm{eV}$ to $30 \mathrm{eV}$. It also shows a $\mathrm{Be}^{+2}$ "plateau" between roughly $30 \mathrm{eV}$ and $12 \mathrm{eV}$, where the wide separation in $\mathrm{K}$ - and $\mathrm{L}$-shell energies Ieaves 
a stable $B e^{t 2}$ plasma over a wide $T$, $\rho$ range, and finally, it shows the recombination of the $L$ shell, from 12 to $0.5 \mathrm{eV}$ (the range 2 to $0.5 \mathrm{eV}$ must be seen in Fig. 3 because it was removed in the Fig. 1 original subspace interface). Now when this shell structure is compared to the unstructured statistical atom (TFCMIX) Isotherms along the $\rho_{0}$ isochore, several interesting features appear. First, for the ideal plasma $(T \geq 300 \mathrm{eV})$, the two theories agree exactly (as expected). But, as the coulomb interactions increase, they start to differ and the $\mathrm{K}$-shell recombination produces a large discrepancy in thermal structure. A moderate agreement is again recovered in the $\mathrm{Be}^{+2}$ plateau (probably fortuitous) until the onset of $\mathrm{L}$-shell recombination and $10 \mathrm{w}-\mathrm{T}$ deviations of the TF model destroy agreement. The net result of this is shown in Fig. 4, where a section of both OCCIP and TFC EOS surfaces has to be removed to accommodate the transition from structured $\mathrm{K}$ shell to pressureionized statistical atoms; a second larger segment is removed to accomodate the L-shell transition. The $\mathrm{Be}^{+2}$ plateau region was directly joined, based on the apparent agreement of the two energy surfaces in the 12 to $30 \mathrm{eV}$ region at $\rho_{0}$. This cholce was not a wise one, as shall be seen.

The final composite-EOS surface in F1g. 4 is substantially represented by primary EOS code ca'culations, with perhaps $10 \%$ of the total $T, \rho$ independent variable range replaced by numerical reconstruction. Along the various subspace interfaces the code to code agreement ranges from quite good to poor. It should be kept in mind here that these primary EOS codes may not be accurate, and a continual program to upgrade exch theoretical area is necessary.

The numerical reconstruction of the interpolation regions is performed in a manner which attempts to satisfy the full set of thermodynamic constraints discussed above. A thermodynamically stable (TDS) method is used to fill the gaps, which ensures that the continuous EOS pressure and energy have positive derivatives $C_{v}$ and $\beta_{T}$. A second process is performed to renormalize the energy surface relative to the pressure surface so that the thermodynamic consistency (TDC) constraint is obeyed. If both these steps are successful, as was the case for $B e$, it appears that the thermodynamic normality (TDN) requirements are also satisfied.

Two operational problems did occur in the TDS-TDC-TDN numerical processing. First, the $\mathrm{L}-\mathrm{V}$ two-phase region had TDC problems, the result of numerical inaccuracies in the GRAY calculation. This will not have a major effect for mist uses of the EOS, 
but calculations which cross the $\mathrm{L}-\mathrm{V}$ two-phase region should be critically examined. Second, when TDC integrat:-ons were performed to correct the energies in the interpolation regions, some TD instability ( $C_{v}$ negative) resulted in the $16-$ to $25-\mathrm{eV}$ range for $\rho>100 \mathrm{Mg} / \mathrm{m}^{3}$. This was a consequence of the direct OCCIP-IFC interface made in the $\mathrm{Be}^{+2}$ plateau reglon where the apparent good agreement in $P$ and $E$ of the two surfaces was not, in fact, adequate. This instability was removed, and the sensitivity of the TDC process to very small defects in the pressure surface was recognized. It should be possible to use this sensitivity to locate regions where model interfaces are not sufficiently smooth and to iterate the interfaceconsistency operations to achieve a satisfactory result.

The final Be EOS energy is shown in Fig. 2. The various regions of special physical interest are indicated. This EOS and the pressure EOS are continuous, stable, consistent (with the exception noted above), and obey the TDN constraints.

\section{EOS Analysis}

The next step in the EOS-generation procedure is analysis of the EOS. The obvious step is a close comparison of the therretical EOS with experimental data. A further useful step is to compare physical-process paths (Hugoniots, isentropes, isobars, etc.) over a large portion of the $T, \rho$ range with other theoretical models.

There is a fairly small amount of high-quality experimental data on $B e$ available for comparison with the theoretical EOS. The most useful data for EOS purposes is single-shockgenerated, high-pressure Hugoniot data. We have chosen the Be data of McQueen et al. from Van Thiel's Compendium of Shock Wave Data. 5 The single-shock data extends up to $885 \mathrm{kbar}(88.5 \mathrm{GPa})$, and a comparison of theory and experiment is shown in Fig. 5. The good agreement between them is expected, in that the CKM region uses a fit to the experimental $u_{s}-u_{p}$ data to define the compression $\left(\rho>\rho_{0}\right)$ portion of the multiphase region. It should be noted, though, that this theoretical Hugoniot is calculated from the final EOS primary data base (PDB) and is exactly the curve a hydrodynamic code would give using this EOS. A different form of the shock-wave data is shown in Fig. 6 (Hugoniot pressure versus density), 


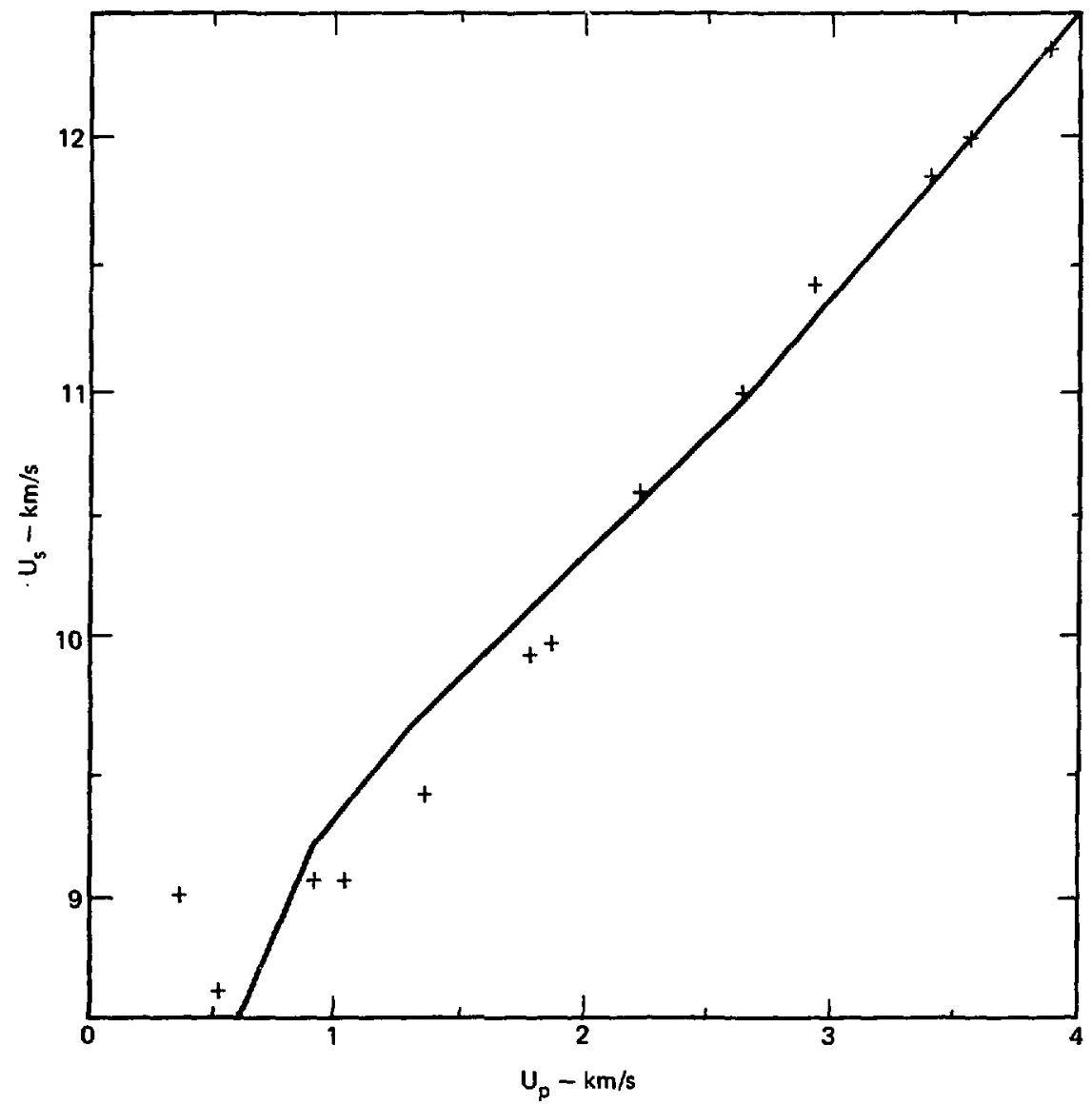

Fig. 5. Comparison of experimental and theoretical shock wave data, $u_{s} v_{s} u_{p}$. The experimental data (*) of McQueen et al is very accurately reproduced by the theoretical Hugoniot (trace). The lowest point is probably an elastic precursor and should not be reproduced by the Hugoniot.

and in this space the agreement between theory and experiment is better. Beryllium shock data even at higher pressures would be desirable to check the theoretical model [885 kbar
(88.5 GPa) only corresponds to a density of $\left.2.7 \mathrm{mg} / \mathrm{m}^{3}\right]$.

A much more demanding test of the theoretical EOS can be made by a comparison with the single- and 


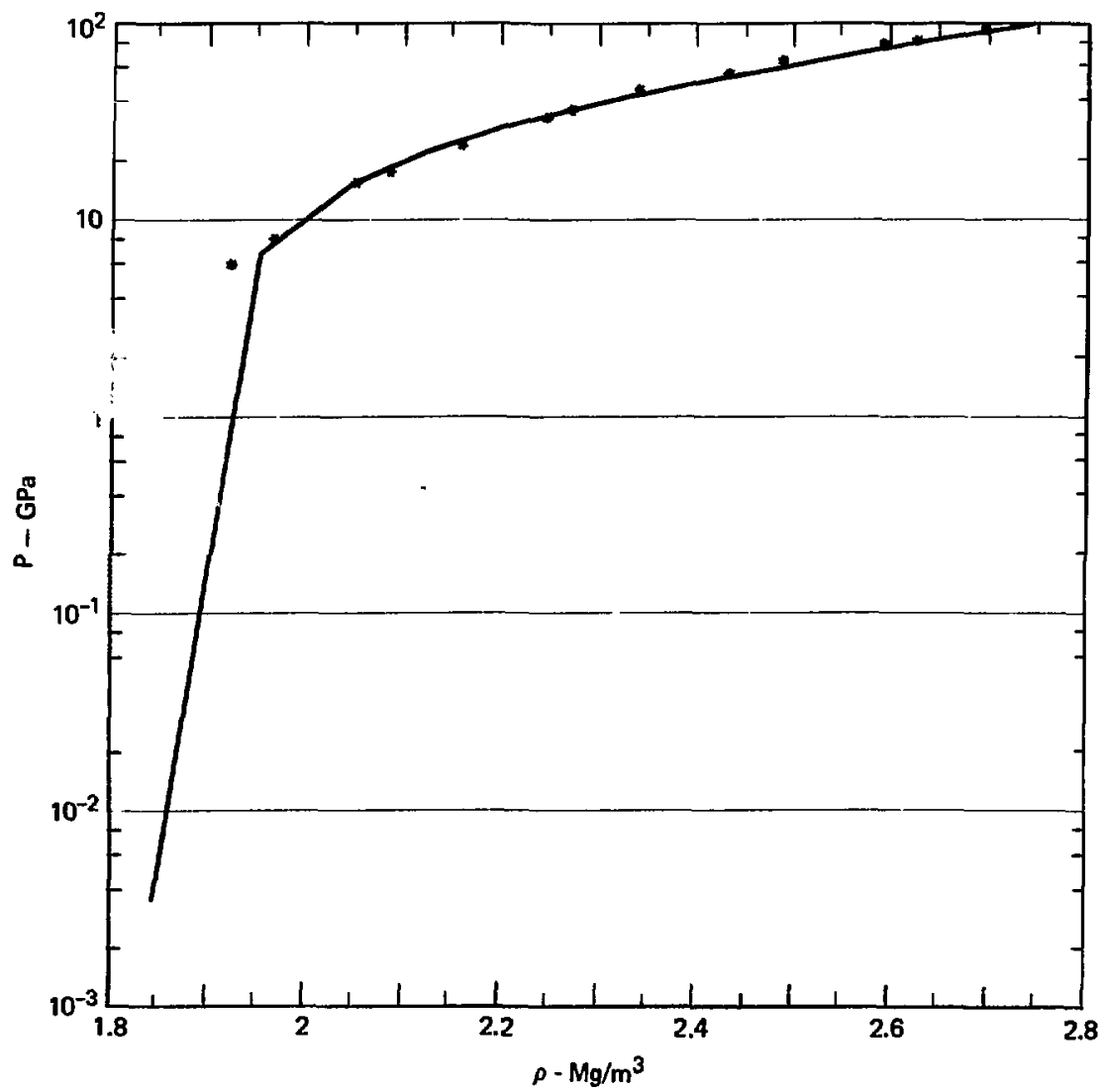

Fig. 6. Comparison of same experimental (*) and theoretical shock Hugoniot loci in pressure and density plane. Deviations in the 300 to 885 kbar range are of the order of a few per cent.

multiple-shock data of Neal. ${ }^{6} \mathrm{~A}$ series of experiments on Be were performed, using colliding shocks to reach the doubly and triply shocked states that lie between the principal
Hugoniot and the principal adiabat. The single-shock pressure achieved was $324 \mathrm{kbar}(32.4 \mathrm{GPa})$, the double shock $788 \mathrm{kbar}(78.8 \mathrm{GPa})$, and the convergent triply shocked state 
1.41 Mbar (141 GPa). The latter two pressures were calculated from the mode1

$$
\gamma_{0}=2 S_{0}-(t+2) / 3,
$$

using $t=0$ (the slater $\gamma$ formulation). These results were compared to the Be theoretical EOS by compul Ing single-, double-, and triple-shock states. As can be seen from Fig. 7, the theoretical $u_{s}-u_{p}$ curves for single, double and triple shocks pass quite close to the single- and doubleshock experimental data, and the error bars for the triple-shock data approach the curve. The same comparison in the pressure-density plane in Fig..8 shows how the double- and tripleshock points lle well below the principal Hugoniot. The substantially lower pressure of the triple-shock experiment relative to the theoretical triple Hugoniot indicates that the rate of decrease of $\gamma$ with volume may be somewhat lower than the value chosen for the GRAY ca!culations.

The foregoing comparisons with experiments in the strongly compressed region of density only give information about the solid Eos model. If high-temperature data is examined, information concerning the melting and liquid behavior of the metal is elucidated. The best high-temperature data for $\mathrm{Be}$ is that discussed by Hultgren et al. 7 They have selected an optimized set of heat-content data extending up to $2200 \mathrm{~K}$, with an extrapolation to $2800 \mathrm{~K}$, primarily based on the work of Kantor et a1. ${ }^{8}$ Comparlson between the enthalpy, $\Delta \mathrm{H}\left(\frac{\mathrm{MJ}}{\mathrm{kg}}\right)$ as a function of $T$ at one atmosphere is shown in Fig. 9. The melting transition in the theoretical model is constrained to occur at the correct temperature; however, the melting density is modeled. Steinberg ${ }^{9}$ has noted a simple relation between the bolling temperature of elemental metals and their liquid density. From this relation, he estimates $\rho_{\mathrm{B}}=1.69$ $\left(\mathrm{Mg} / \mathrm{m}^{3}\right)$ for $\mathrm{Be}$. The theoretical nodel predicts $\rho_{B}=1.70$ and is in good agreement.

A further comparison in the high $T, r$ expansion $\left(\rho<\rho_{0}\right)$ part of the multiphase $T$, $\rho$ range is given in Fig. 10. Here the melting curve for bcc Be is plotted from data in the compendium of Young. 1 There is only moderate agreement between theory and experiment, since the theoretical melting temperatures are about $100 \mathrm{~K}$ low in the range $35-60 \mathrm{kbar}(3.5-6.0 \mathrm{GPa})$. It should be kept in mind that, while errors of this magnitude are not desirable, the theoretical model is reasonably close to the true physical conditions.

A final overall comparison of the high-temperature plane can now be made by looking at three key high-T conditions: the melting, boiling and critical points. The first two conditions have been discussed; the critical 


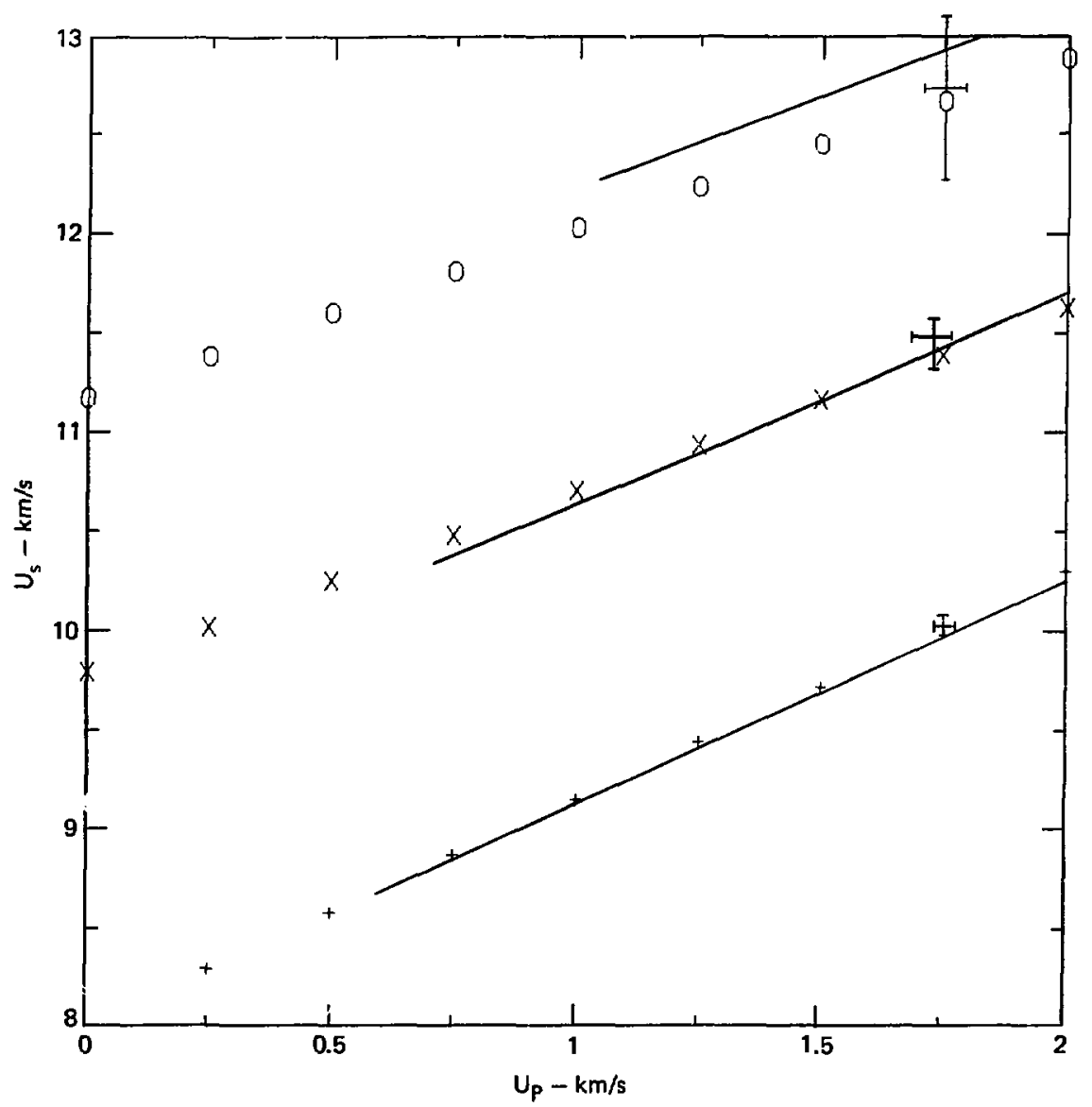

Fig. 7. Comparison of multiple shock experimental results of Neal with theoretical multiple Hugoniots calculated from the theoretical Be EOS. The experimental data points are shown $(+)$ for single, double and triple shocks. Neal's $u_{s}-u_{p}$ curves calculated from a Mie-Grüneisen EOS vith a Slater $\gamma$ are also shown $(t, x, 0)$, while the theoretical multiple Hugoniot paths appear as traces. All are in reasonable agreement with the experimental points.

point for Be is unknown; however, using a method developed by Young and Alder ${ }^{10}$ the critical conditions can be estimated. Using a Van der Waals model, a hard-sphere expression from Monte Carlo computer experiments, and an effective hard-sphere estimate for the metal, Young estimates the critical conditions given in Table 3 . It is clear from the table that the 


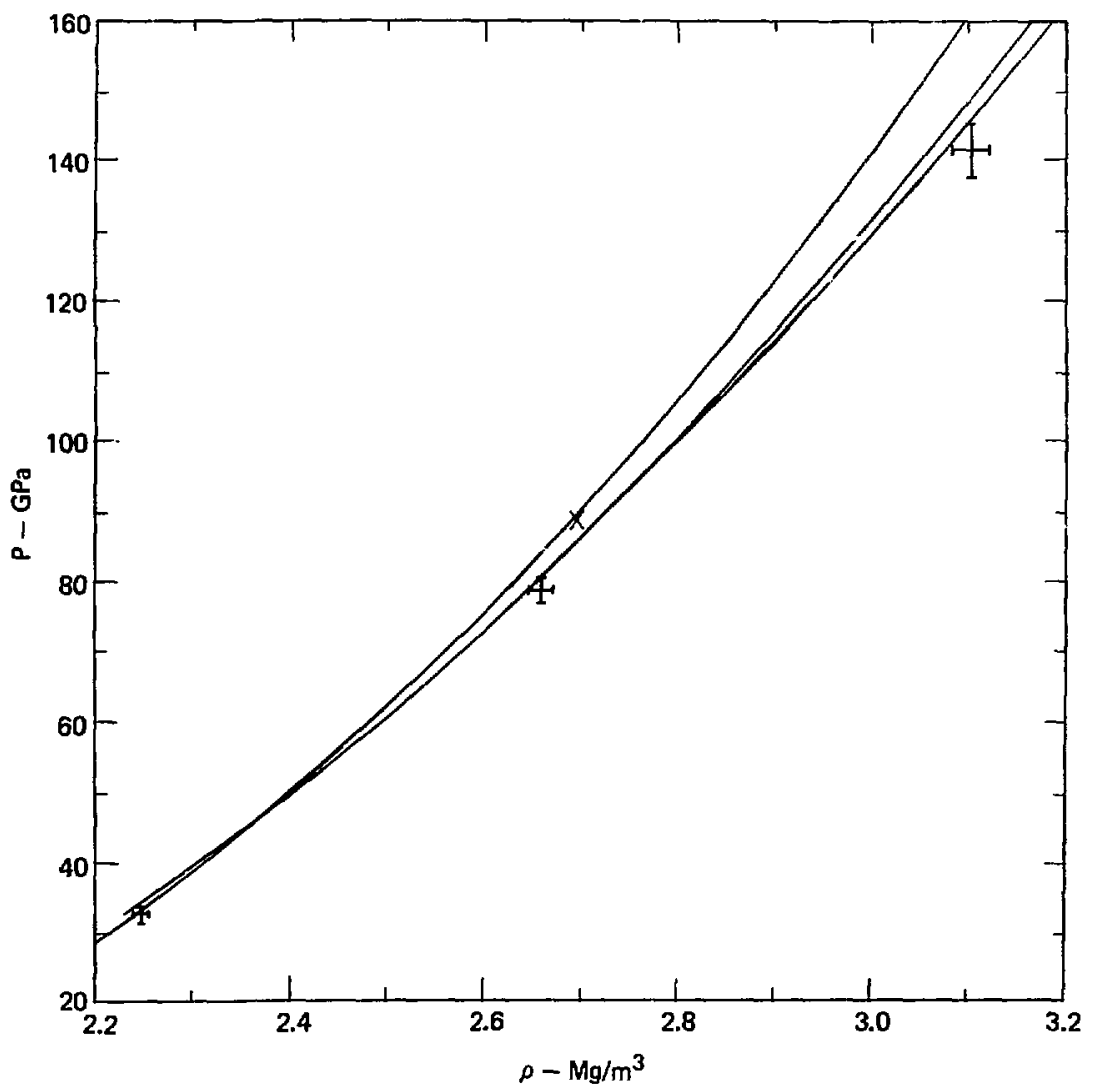

Fig. 8. Comparzson of shock Hugoniot data with theoretical Hugoniots in the P- $\rho$ plane. Neal's $32.4 \mathrm{GPa}$ point, and the $88.5 \mathrm{GPa}$ single shock point of McQueen et al hoth Ife on the principal Hugoniot. Neal's double shock point lies just below (within experimental erroc) the reflected shock Hugoniot at $78.8 \mathrm{GPa}$. The triple shock point lies well below the triple shocked Hugoniot, but the large error bars make this point less critical for theoretical comparisons.

model matches the experimental data well near melt, but is less than satisfactory at the lower densities. This failure to match bofling and critical conditions is a consequence of the inadequacy of the simple hardsphere fluid model in representing the true $\mathrm{Be}$ dense-fluid regime. A close fit to either the boiling or critical conditions could have been 


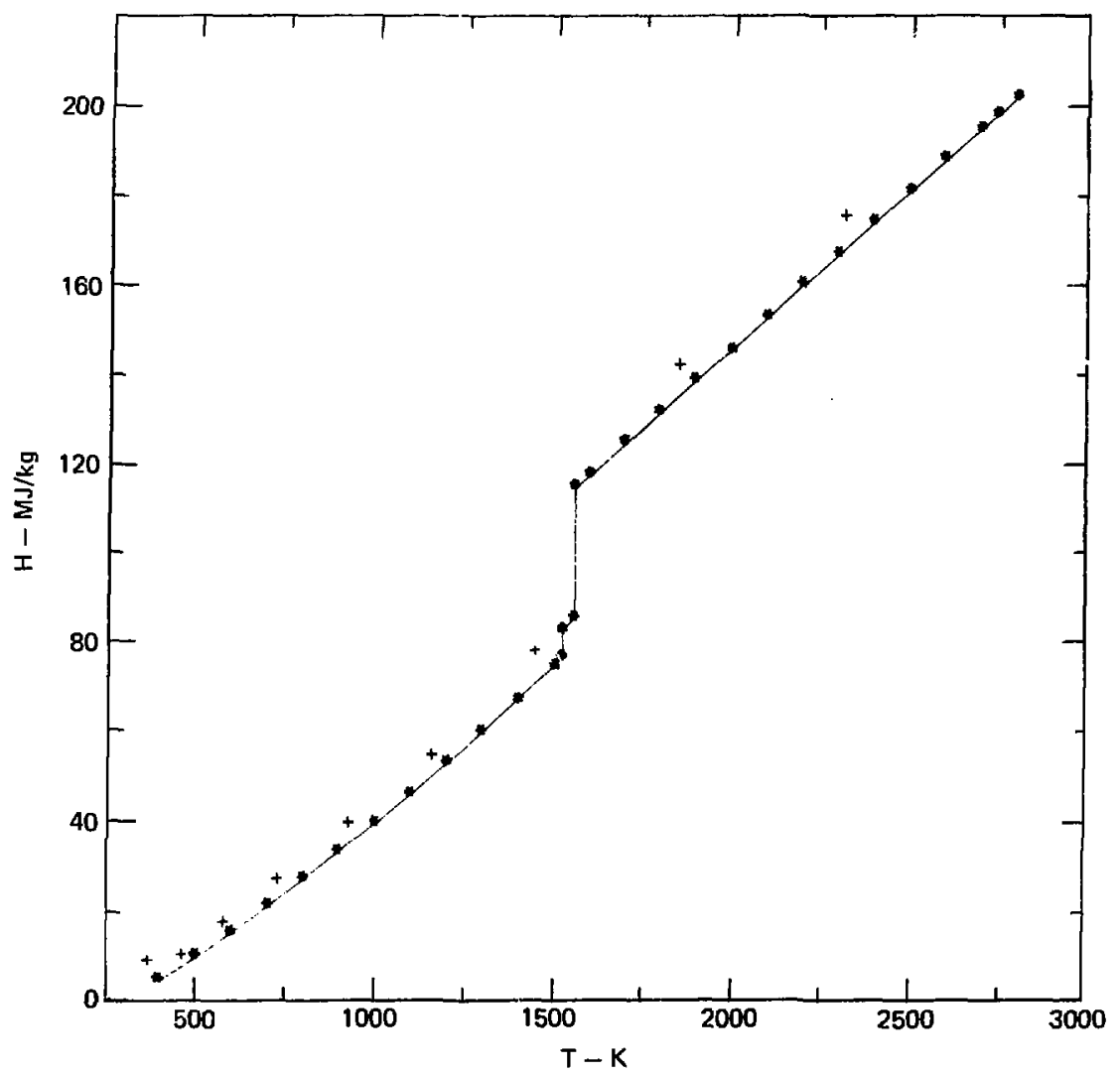

Fig. 9. The high temperature experimental data for heat content, $\Delta \mathrm{H}\left(\mathrm{Mbar} \cdot \mathrm{cm}^{3} / \mathrm{g}\right)$ versus temperature $(\mathrm{K})$ at 1 atmosphere (101 $\mathrm{KPa})$, taken from Hultgren et a1 (trace), compared to the theoretical 1 bar (101 $\mathrm{KPa})$ isobar. The theoretical isobar exactly parallels the experimental one, with the melting transition occurring at $1551 \mathrm{~K}$. The theoretical $\Delta \mathrm{H}$ is equivalent to the solid-solid $(\alpha-\beta)$ plus melting enthalpy change, since the theoretical model does not attempt to mode1 the $\alpha-\beta$ transition.

achieved by appropriate adjustment of $E_{\text {coh }}$ A choice of $E_{\text {coh }}=0.0377$ $\mathrm{MJ} / \mathrm{mole}$ (the true experimental value) would have exactly matched the boiling-point conditions, but with a resulting $\mathrm{T}_{c}$ about two times larger than Young's estimate. Likewise, a much lower $\mathbf{E}_{\text {coh }}$ would give an exact match to critical-point estimates, at the cost of an even lower $T_{b}$ than 


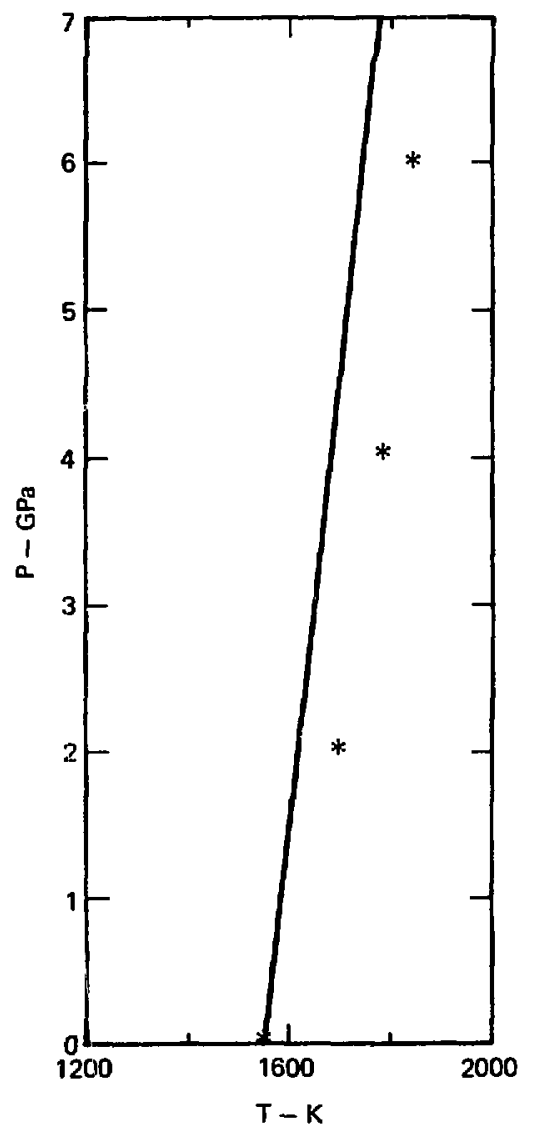

that given in Table 3. The choice was made to adjust $E_{\text {coh }}$ to come as close as possibje to both $\mathrm{T}_{\mathrm{b}}$ and $\mathrm{T}_{\mathrm{c}}$. The correct alternative is to develop a more sophisticated dense-fluid model to allow accurate theoretical prediction of the entire 1iquid-vapor region.

All the foregoing discussion of experimental-theoretical agreement is limited to $\mathrm{T} \leqslant 0.25 \in \mathrm{V}$ (for actuul data), a very low value compared to the full range of the EOS surface. The rest of the $T, \rho$ range is devoid of any experimental checks. In the IE region, the Be ionization potentials are experimentally known, so the theoretical model is based on experiment as long as the ideal gas assumption remains valid, say, for $\rho<10^{-2} \mathrm{Mg} / \mathrm{m}^{3}$, at $\mathrm{T}>10 \mathrm{eV}$ and $\rho<10^{-1}, \mathrm{~T}<100 \mathrm{eV}$.

Fig. 10. The melting curve for Be. $T_{m}$ versus $P_{m}$ (bce to liquid is shown for theoretical (trace and experimental ( $*$ ) data. The experimental values are taken from Young's compendium, and extend to about 60 kbars $(6 \mathrm{GPa})$.

Table 3. High temperature experimental data compared to the theoretical Eos.

\begin{tabular}{|c|c|c|c|c|c|c|}
\hline \multicolumn{3}{|c|}{ Boiling } & \multicolumn{2}{|c|}{ Melting } & \multicolumn{2}{|c|}{ Critical Point } \\
\hline Exper & mental & Theoretical & Experimental & Theoretical & Estimated & Theoretical \\
\hline$T_{B}$ & $3090 \mathrm{~K}$ & $2251 \mathrm{~K}$ & $T_{m} 1551 \mathrm{~K}$ & $1551 \mathrm{~K}$ & $T_{c} 7474 k$ & $8484 \mathrm{~K}$ \\
\hline$D_{b}$ & $1.366 \mathrm{Mg} / \mathrm{m}^{3}$ & 1.495 & $\rho_{\mathrm{m} \cdot} 1.69$ & 1.70 & $\rho_{\mathrm{c}} \quad 0.493$ & 0.606 \\
\hline$P_{b}$ & $\begin{array}{l}1 \text { atm. } \\
\text { (101) KPa) }\end{array}$ & $\begin{array}{l}1 \mathrm{~atm} . \\
(101 \mathrm{kPa})\end{array}$ & $\mathbf{T}_{\mathrm{m}}^{\circ}-$ & $19^{\prime} \mathrm{r} \mathrm{K}$ & $\begin{array}{ll}P_{c} & 1.22 \times 10^{-2} \mathrm{Mbar} \\
& (1.22 \mathrm{GPa})\end{array}$ & $\begin{array}{l}1.71 \times 10^{-2} \\
(1.71 \mathrm{GPa})\end{array}$ \\
\hline $\mathbf{E}_{\text {coh }}$ & $\begin{array}{l}3.2 \frac{\mathrm{Mbar}-\mathrm{cm}^{3}}{\mathrm{~mole}} \\
\left(0.32 \frac{\mathrm{MJ}}{\mathrm{mol}}\right)\end{array}$ & $\begin{array}{l}2.4 \frac{\mathrm{Mbar}-\mathrm{cm}^{3}}{\mathrm{~mole}} \\
\left(0.24 \frac{\mathrm{MJ}}{\mathrm{mol}}\right)\end{array}$ & & & & \\
\hline
\end{tabular}


Similarly, at extremely high pressures, say $\mathrm{P}>1000$ Mbar (100 TPa), the TF models are strongly based on simple extensions of Fermi-Dirac electron statistics, also experimentally known. But it is evident that a wide gap from 1 Mhar to 1000 Mbar (0.1 to $100 \mathrm{TPa}$ ) or from $0.2 \mathrm{eV}$ to $100 \mathrm{eV}$ is totally based on the complicated theoretical models or on numerical thermodynamic approximations.

One final set of comparisons can be made to demonstrate the range of theoretical variance occurring in two different models. A well established theoretical model for $\mathrm{B} e$ is the work of Cowan, ${ }^{11}$ who developed theoretical EOS for elements using finitetemperature, Thomas-Ferni-Dirac (TFD) physics for the electron gas, with ideal-gas ideal-solid ion contributions. These EOS were further corrected by additional information from high-pressure shock data, limited to data obtained prior to 1957. Instead of the formidable task of comparing the full pressure and energy surfaces, it is more instructive to analyze certain key physical-process paths, such as Hugoniot paths and isentropic release paths. A comparison of the Cowan TFD-based Be theoretical Hugoniot with the correspondi:1g track computed from the new Be EOS is shown in Fig. 11. The two theories agree well within the high-T plasma limit $[\mathrm{P}>5000$ Misar (500 TPa) $\mathrm{T}>1 \mathrm{keV}$ ] but differ significantly almost everywhere else. In the experimental regime $[P \leqslant 1$ Mbar $(100$ GPa $)]$, the older EOS does not have a defined Hugoniot: in the figure, the lowest Hugoniot pressure calculable is 1.55 Mbar (155 GPa), which results from its original design as a very highpressure equation of state. In the 1-1000 $\mathrm{Mbar}(0.1-100 \mathrm{TPa})$ intermediate region the major feature is the appearance of shel1-structure (QM) ionization equilibrium, which causes the principal Hugoniots to differ even qualitatively. The TFD Hugoniot is relatively smooth and nonotonic, averaged through the four ionization levels; the she11-structure Hugoniot exhibits two large loops, the lower one [ 4 to 30 Mbar $(400$ to $300 \mathrm{GPa})$ ] occurring as the L-shell electrons are pressure iozized. There is a minimum between the shells, corresponding to the $\mathrm{Be}^{+2}$ plateau, then again a second loop, from 50 to $3000 \mathrm{Mbar}$ (5 to 300 $T P a)$, as the $\mathrm{K}$-shell electrons are ionized, Be is a member of a small class ố low-Z materials He, Li, Be and perhaps $B$, where the nearly equal population of the $K$ and $L$ shells gives comparable shell structure effects on the Hugoniot. The high-z elements have pressure ionization effects smeared out over many electron shells, so their structure is much 


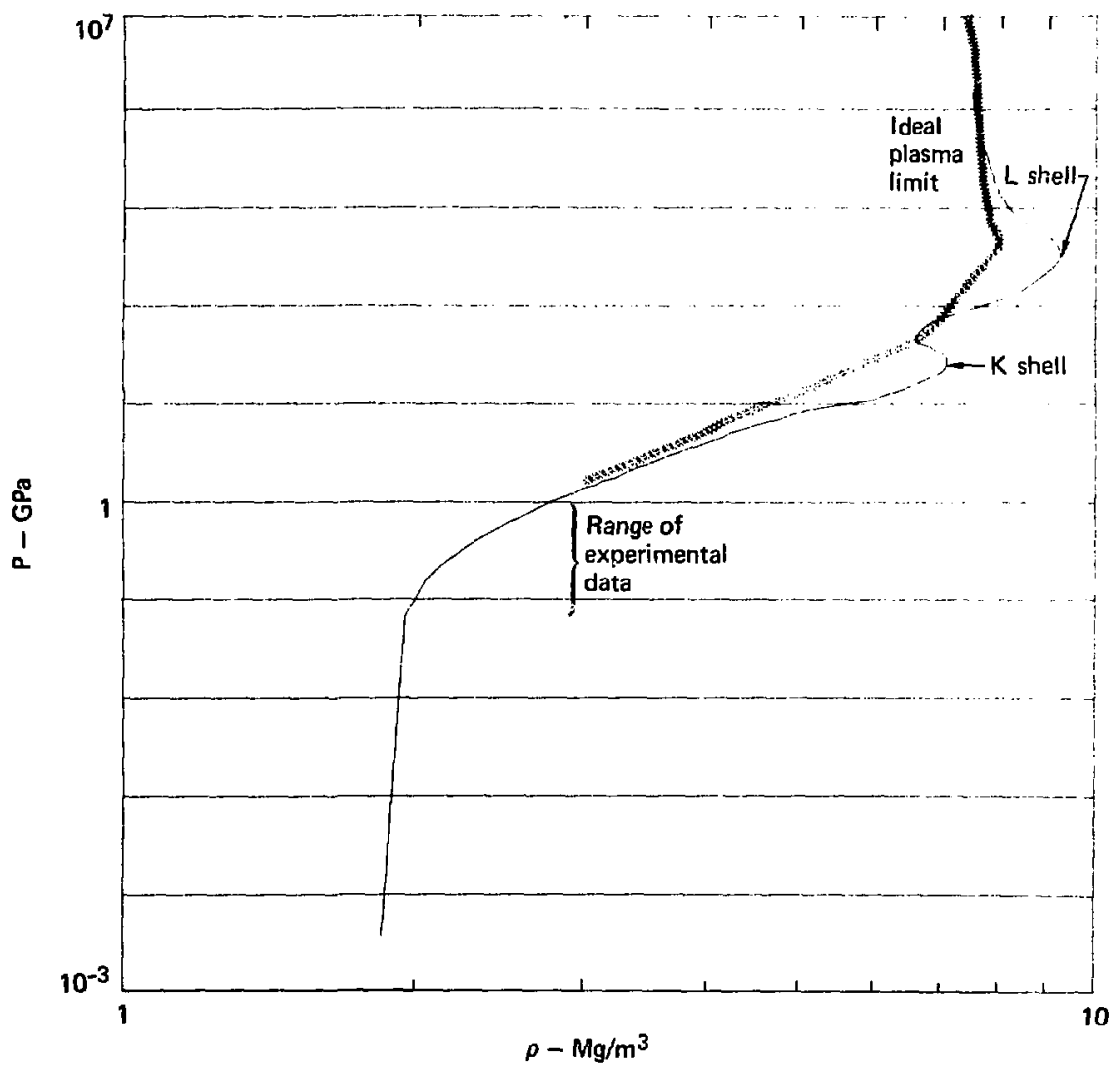

Fig. 11. The principal Hugoniots computed from two theoretical models. The TFD-based EOS of Cowan (***) shows typical smooth statistical atom behavior, while the new EOS goes from good agreement with shock data for $P<1$ Mbar (100 GPa), through two shell structure pressure ioxlzations corresponding to the $K$ and $L$ shells.

closer to a smooth statistical atom model. Even for elements with $Z$ of 6 to 12, the larger population of the L shell results in a smoothed I-shell structure, with only the $\mathrm{K}$ shell appearing in the pronounced manner seen here.
A second type of physical-path comparison is the release isentrope, where material is allowed to isentropically expand from some initial state. This is shown in Fig. 12 for the two theoretical EOS's. The initial states are $0.1,1,10,100$ and 


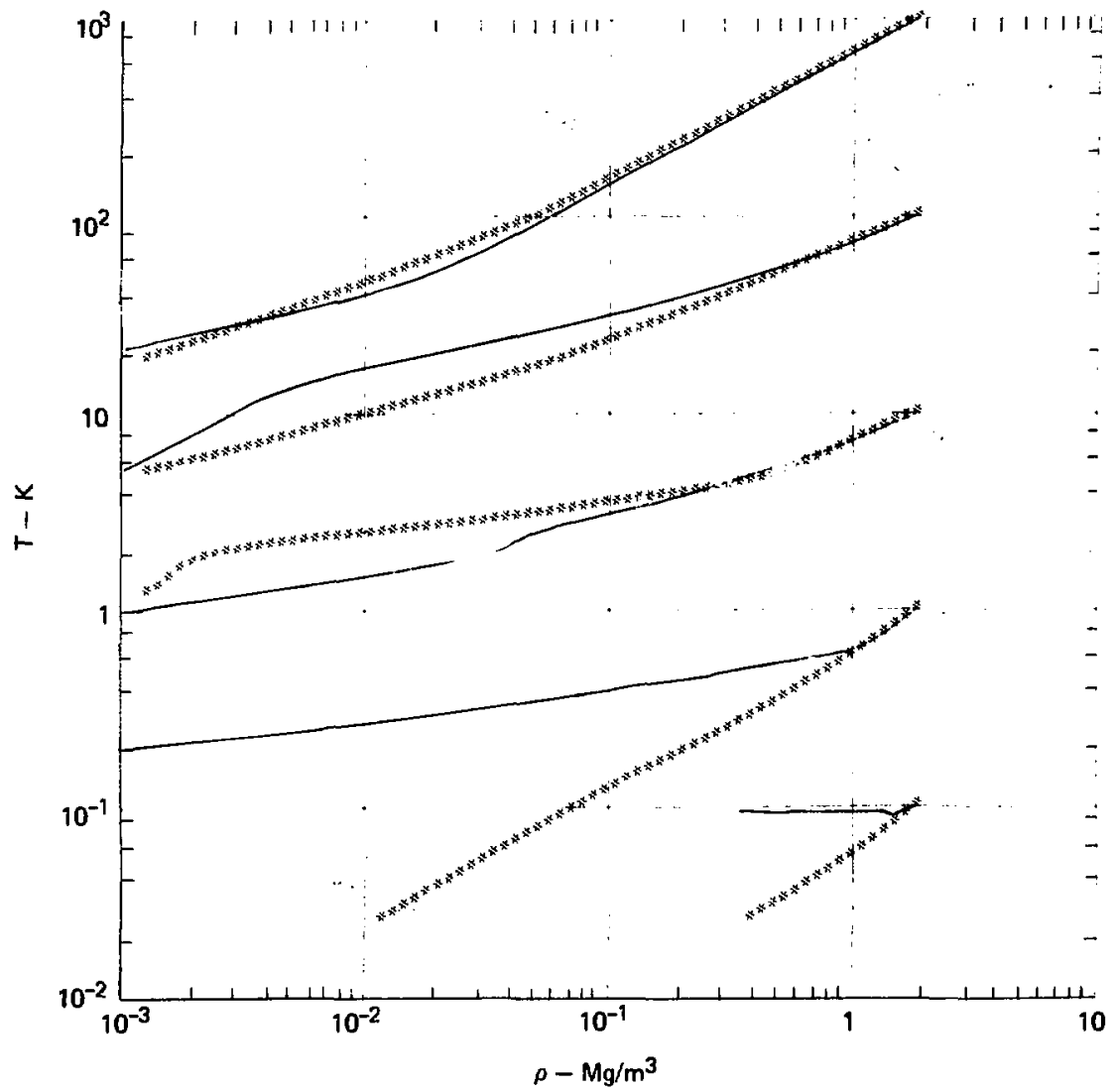

Fig. 12. Release isentropes in $T, \rho$ space calculated from TFD $(* * *)$ and the new Be EOS (trace). Significant differences exist in the expansion paths for all initial states from 0.1 to $1000 \mathrm{eV}$. The 10,100 , and $1000 \mathrm{eV}$ paths pass through the low density thermal ionization regions, causing differences with statistical atom predictions, while the low $T(0.1,1 \mathrm{cV})$ paths pass through the dense neutral fluid and multiphase regions.

$1000 \mathrm{eV}$, at $\rho=\rho_{0}$. In ali the high-T low $\rho$, the theoretical assumption of cases, deviations between the TFD and shell-structure EOS are noted, including temperature differences as large as $80 \%$. At these high $T$ and an ideal partial-ionization region is quite reasonable so these differences are probably real. This means equivalent differences will exist for 
$P$ and $E$ along these same tracks. Perhaps the major significance for these differing $T$, $\rho$ paths lies not in the different hydrodynamic states attained, but in the use of the $T$ values for calculating strongly $\mathrm{T}$-dependent transport properties, such as opacity.

In the low-temperature regime ( $T \leq 1$ eV) the release isentropes differ tremendously, as would be expected. The TFD model of this regime, designed for very high pressure, yields a simple plasma-like release curve, new EOS gives very different behavior; the 1-eV isentrope stays in the fluid regime, passing just above the critical point. The $0.1 \mathrm{eV}$ release path passes through melt and then into the two-phase region, where $T$ is almost constant.

With the prospect of new techniques while the multiphase structure of the using laser-generated shock wives to reach much higher pressures on the principal Hugoniot, ${ }^{12}$ it might be interesting to see what shock

pressures would be required to melt $\mathrm{Be}$ and to reach the fully liquid state, according to the present theoretical model. These values can be seen in Figs, 13 and 14 from the theoretical Hugoniot, and the melting and freezing curves. It appears that a single-shock pressure of about $1.95 \mathrm{Mbar}(195 \mathrm{GPa}$ ) is needed to melt Be on the Hugoniot. This occurs at a temperature of $0.4 \mathrm{eV}$. To pass through the two-phase region and reach the fully liquid state, a 2.5 Mbar (250 GPa) shock is required, corresponding to $0.45 \mathrm{eV}$. It is quite possible that such shock strengths can readily be achieved by the existing large laser systems.

\section{Summary and Conclusions}

A complete theoretical EOS for Be has been generated, ranging from $10^{-3}$ to $2000 \mathrm{Mg} / \mathrm{m}^{3}$ and from 0.02585 to $2.5 \times 10^{5} \mathrm{eV}$. The basic theoretical models which have been combined to treat this material are a coulombcorrected ionization equilibrium theory, OCCIPITAL; the finitetemperature, Thomas-Fermi-Kirzhnits electron model plus BST ion-fluid contributions for the high-pressure, high-density regime, and the GRAY multiphase model, combining the MieGruneisen solid, Lindemanu law melting, scaling model liquid and hard sphere fluid, The resultant composite EOS is thermodynamically stable and consistent, and is very successful in matching the high-pressure shock Hugoniot data, both single and multiple 


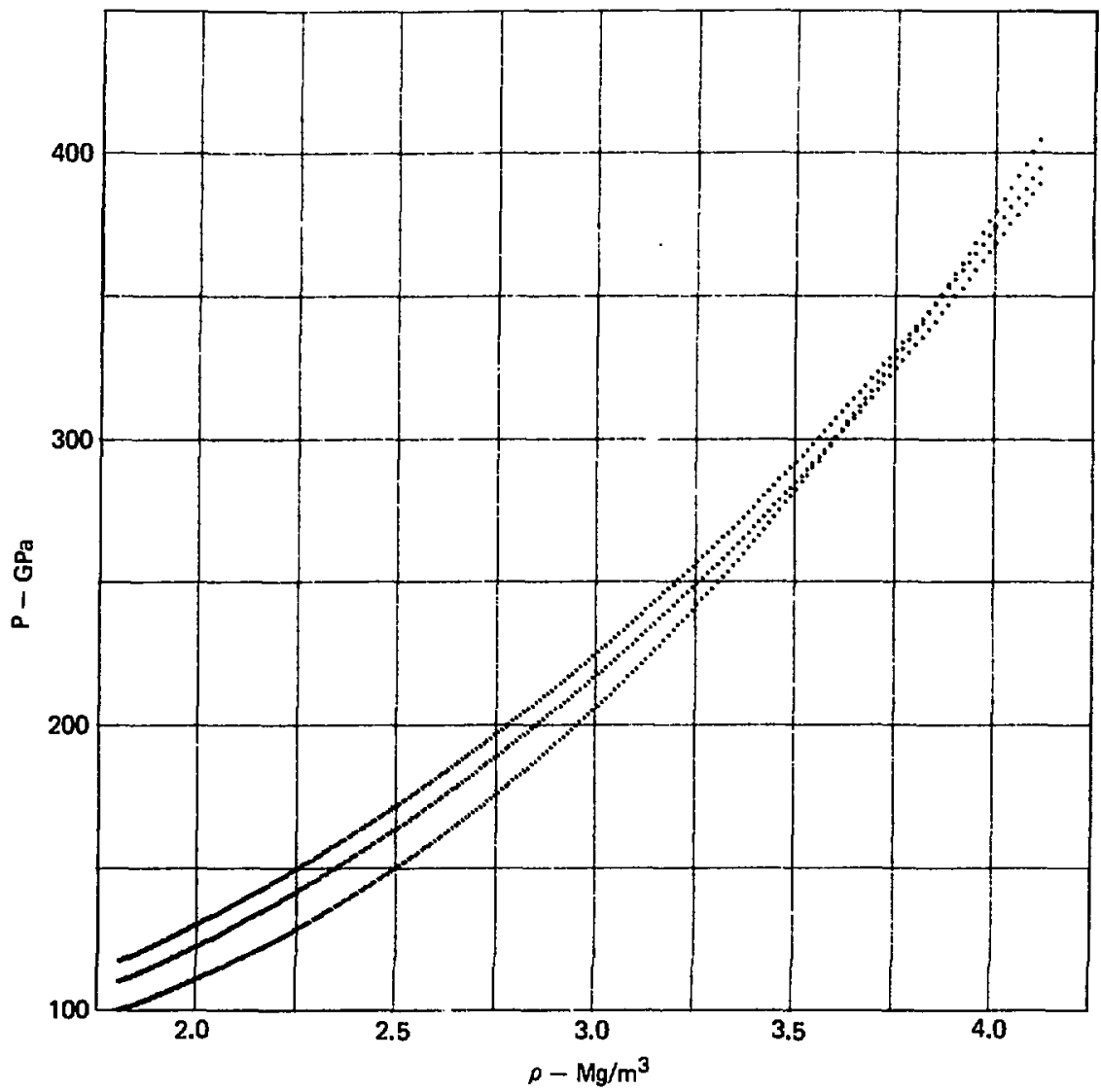

Fig. 13. The principal Hugoniot, melting (solidus) and freezing (1iquidus) loci with pressure as a function of density calculated from the Be theoretical EOS. According to this work, the melting transition in Be could be observed at 1.95 Mbar ( $195 \mathrm{GPa}$ ), and complete transition to the liquid state would be achieved by 2.5 Mbar (250 GPa).

shock. It is only partially successful melting experimental data, and only in treating the high temperature data, roughly matches the boiling and esagreeing well with heat content and timated critical conditions. When 


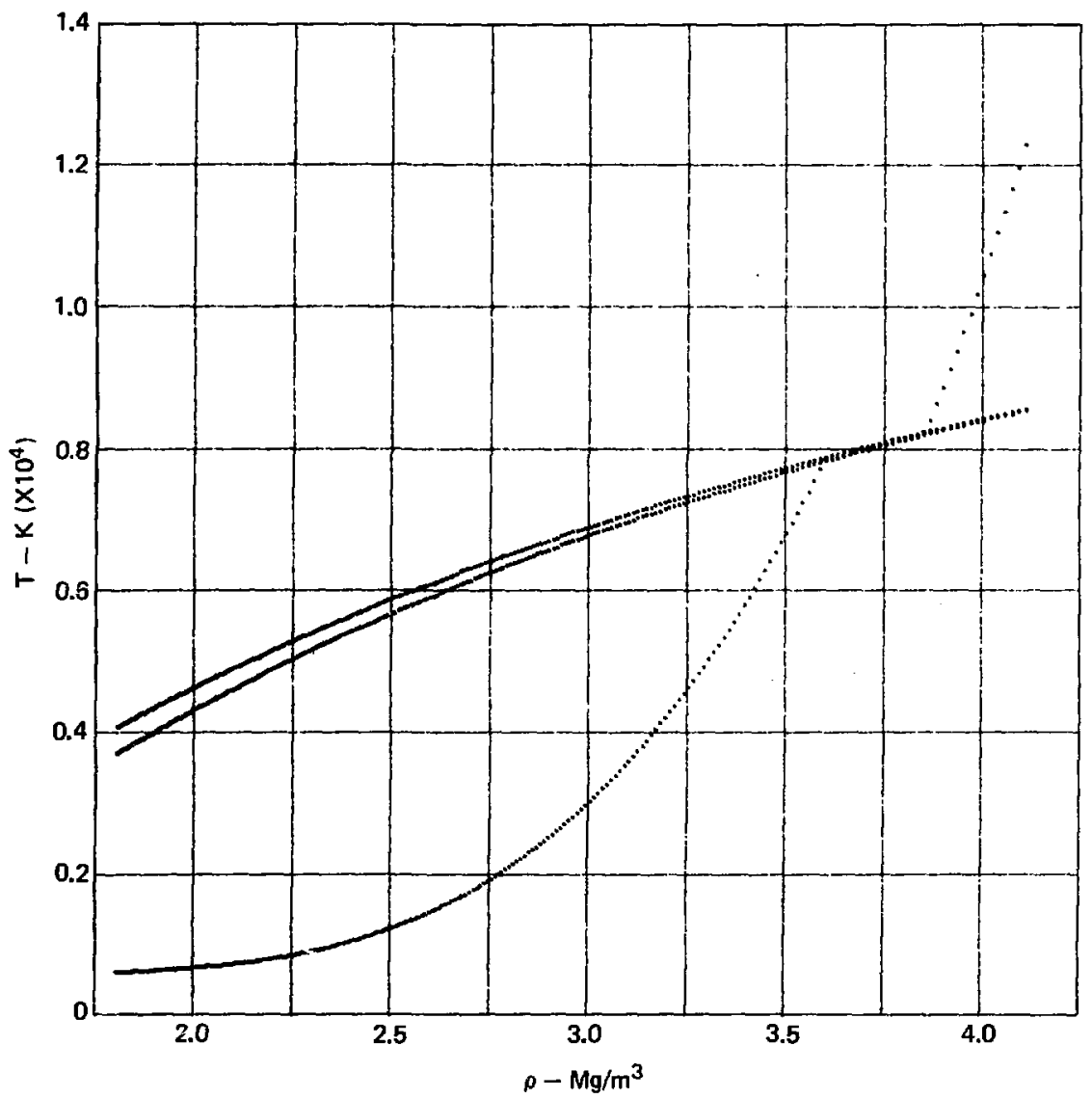

Fig. 14. The corresponding Hugoniot, melting and freezing 10ci in temperature density space, showing the onset of melting at $0.4 \mathrm{eV}(\eta=1.63)$, and complete liquefaction at $0.45 \mathrm{eV}(n=1.87)$.

compared to older pure statistical atom models, substantial differences are observed.

The basic features of the new Be EOS are:

(1) Good agreement overall with a wide range of experimental data, both high pressure and high temperature.

(2) The appearance of strong shell structure effects in the EOS surface, which have significant 
effects on both shock compression and isentropic expansion from high $\mathrm{T}$ states.
(3) A complete multiphase model of the $S-L-V$ region, including melting and evaporation.

\section{Acknowledgments}

We would like to thank R. Grover and D. Young for theoretical analysis and helpful. comments. J. Shaner and
D. Steinberg were helpful in aiding us in obtalning some of the experimental data.

\section{References}

1. D. A. Young, Phase Diagrams of the Eiements, Lawrence Lfvermore Laboratory, Rept. UCRL-51092 (1975).

2. C. A. Rouse, Astrophys. J. 136, 599, 636, 665 (1962).

3. S. L. McCarthy, The Kirzhnits Corrections to the Thomas-Fermi Equation of State, Lawrence Livermore Laboratory, Rept. UCRL-14364 (1965).

4. E. Royce, GRAY, A Three-Phase Equation of State for Metals, Lawrence Livermore Laboratory, Rept. UCRL-51121 (1971).

5. M. Van Thiel, Comperidium of Shock Wave Data, Lawrence Livermore Laboratory, Rept. UCRL-50108 (1967).

6. T. Nea1, Reflected Rarefactions, Double Regular Reflections, and Mach Waves in Aluminum and Beryzlium, LA-UR 75-934 (1977).

7. R. Hultgren, P. Desai, D. Hawkins, M. Gleiser, K. Kelley, and D. Wagman, Selected Values of the Thermodynomic Properties of the Elements, American Society for Metals, Metals Park, Ohio (1973).

8. P. B. Kantor, R. M. Krasovitskaya, and A. N. Kisel, Phys. MetaZs MetalZog. 10 (6), 42-4 (1960).

9. D. J. Steinberg, "A Simple Relationship Between the Temperature Dependance of the Density of Liquid Metals and their Boiling Temperatures," MetalZurgical Trans. 2, 1341-1343 (1974).

10. D. A. Young, and B. J. Alder, "Critical Point of Metals from the Van der Waals Mode1," Phys. Rev. A 3, 364-371 (1971).

11. R. D. Cowan, Thomas-Fermi-Dirac Equation of State, LA-2124, (1957).

12. R. J. Trainor, LASNEX, Study of Laser-Initiated Shock Compression in Planar Targets, Lawrence Livermore Laboratory, Rept. UCRL-50028-77-1, 1977. 